\title{
10 Zur zweiten Meta-Frage: MSI, RTI und HSI - die erste Revolution
}

L'essentiel est invisible pour les yeux.

Antoine de Saint-Exupéry, Le Petit Prince (1943)

\subsection{Die Güte früherer Textausgaben}

Man könnte vermuten, dass für alle bisher aufgerollten Papyri halbwegs akzeptable Ausgaben vorliegen und allenfalls noch Verbesserungen im Detail zu erzielen sind. Dies ist erstaunlicherweise nicht der Fall. Viele nicht in der Gigante-Ära neuedierten Werke wurden im späten 19. oder frühen 20. Jahrhundert auf Basis der collectio altera bzw. der Neapolitanischen Disegni und Oxforder Disegni ediert, oft ohne (gründliche) Autopsie. Die Disegni haben aber in vielen Zeilen schlichtweg überhaupt keinen Text, obwohl im Original Buchstaben lesbar sind. Ferner sind etliche gewagte Ergänzungen früherer Editionen mit einem einfachen oder genaueren Blick auf das Original zu falsifizieren. Dennoch wurden sie, wenn das Griechische akzeptabel war und der Sinn „gefiel“, auch in neueren Ausgaben oft „durchgeschleppt“ und die Papyrusspuren oft (unbewusst) unter der Losung in dubio pro libertate gewünschten Lesungen angeglichen; ferner waren viele Herausgeber ambitioniert, möglichst viel Text zu lesen. Blank (2007) beschrieb die Situation wie folgt:

Re-editions of Herculaneum texts have a tendency to intervene in their predecessors' work only where the text seems unsatisfactory, leaving it alone when its grammar and sense seem smooth: editors tend to make controlli. But the early editions were often made without the benefit of consulting the papyrus, and the editors were often less inhibited about making changes in the text of apographs than they might have been in the text of the papyrus itself. One conjecture called forth others in its neighbourhood, and many passages began to look sound....the editor was not questioning the text, which seemed unobjectionable on the printed page, and the microscope was used to remove dots - no one wants to have seen less than one's predecessors, except where the papyrus has suffered in the interim. Editors should be on their guard, therefore, to begin with a text scrubbed of conjectures and to read every letter in their texts carefully in the original ... challenge their predecessors, more than confirm them. ${ }^{113}$

113 Blank, „Life of Antiochus“ (wie Anm. 94), 93. 
Ebenso gravierend ist der Umstand, dass die Herausgeber bis 2000 oftmals mit dem menschlichen Auge (bzw. Mikroskop) auch in unzerstörten Passagen keine Tinte erkennen konnten, sondern nur einen schwarzen, verkohlten Untergrund sahen und dementsprechend keinen Text transkribieren konnten - da das Geschriebene vermeintlich für immer verschwunden war.

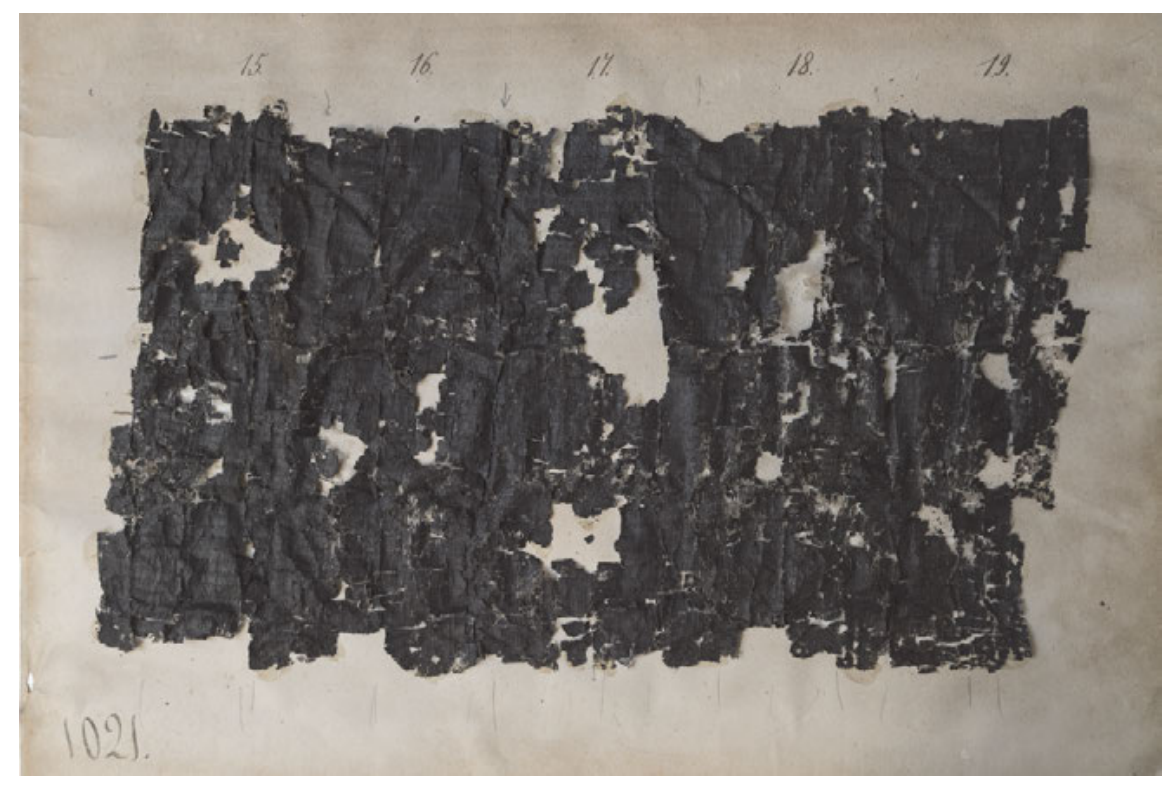

Abb. 28: Bild mit „normaler“ Kamera - cornice 4 (=Kol. 15-19) von PHerc. 1021 (Philodems Index Academicorum)

\subsection{Multispektralbilder (MSI: 1999-2002)}

Um die Jahrtausendwende kam es mit den sogenannten Multispektralbildern (MSI-Multispectral Images) zu einer ersten „epochalen“ Umwälzung in der Herkulanischen Papyrologie. Zwischen 1999 und 2002 wurden von einem Team der Brigham Young University (BYU-Utah/USA) um David Seely und Steven Booras von der gesamten Herkulanischen Sammlung „multispektrale“ Aufnahmen gemacht. ${ }^{114}$ Diese MSI erfassen Licht jenseits der Wellenlängen, welches das

114 Steven Booras und David Seely, „Multispectral Imaging of the Herculaneum Papyri,“ CErc 29 (1999): (95-100); Agathe Antoni/Steven Booras/Gianluca Del Mastro/Roger Macfarlane, 
menschliche Auge wahrnehmen kann, und zeigen somit Text, den man mit dem bloßen Auge nicht oder nur schwer erkennen kann. Der Ausdruck Multispektralbilder ist für die Bilder der Herkulanischen Papyri dahingehend zu präzisieren oder zu relativieren, dass von der Sammlung aus zeitökonomischen Gründen - bis auf wenige Ausnahmen - keine echten Multispektralbilder gemacht wurden, sondern lediglich Bilder im Nahinfrarotbereich (950 nm). ${ }^{115}$ In diesem Bereich ist der Kontrast zwischen Tinte und Papyrus am stärksten. Manche Papyri, auf denen man bis dato mit dem bloßen Auge nichts lesen konnte und die folglich unter „non leggibile“ katalogisiert waren, ließen plötzlich auf den digitalen Multispektralbildern deutliche Buchstaben und zusammenhängende Textabschnitte erkennen. Es kursiert die schöne (und wahre) Anekdote, dass Papyri, für die man vormals lateinischen Inhalt angenommen hatte, sich als griechische Werke entpuppten, während man andere Fragmente verkehrtherum gehalten hatte, weil auf ihnen unter dem natürlichen Licht so wenig lesbar war. ${ }^{116}$ An vielen Stellen helfen die MSI die ungewissen Spuren des Originals zu verstehen oder einzuordnen - manche Stellen wurden durch die MSI überhaupt erst lesbar, viele Stellen besser lesbar. Je nach Karbonisationszustand des Papyrus entfalten die MSI unterschiedliche Tragweite: Für Papyri, welche bisher schon mit den Augen problemlos gelesen werden konnten, waren die MSI textuell freilich nicht oder nur unwesentlich hilfreich, wohingegen der Text ,dunkler“ Papyri oft erhellt oder in einigen Fällen im wahrsten Wortsinne erst „,ans Licht gebracht“ wurde. ${ }^{117}$ Ein weiterer, nicht zu unterschätzender Vorzug der

„Update Report on the Use of the Multi-spectral images of the Herculaneum papyri,“ in Proceedings of the XXIV International Congress of Papyrology, Helsinki 1 -7 August 2004 (Band 2) (hg. von Jaako Frösén/Tina Purola/Erja Salmenkivi; Helsinki, 2007), (579-586). Stephen Bay/Gregory Bearman/Roger Macfarlane/Thomas Wayment, „Multi-Spectral Imaging vs. Monospectral Infrared Imaging,“ ZPE 173 (2010): (211-221); Stephen Bay/Gregory Bearman/Roger Macfarlane/Thomas Wayment, „Exploring the Limitations and Advantages of Multi-Spectral Imaging in Papyrology: Darkened, Carbonized, and Palimpsest Papyri,“ in EIKONOPOIIA: Digital Imaging of Ancient Textual Heritage, Proceedings of the International Conference, Helsinki, 28-29 November, 2010 (hg. von Vesa Vahtikari/Mika Hakkarainen/Antti Nurminen; Helsinki, 2011), (107-121).

115 Die Bilder können heute auf CD-ROM in der Officina dei papiri konsultiert werden und ggf. bei Roger Macfarlane für bestimmte Papyri angefordert werden.

116 Vgl. die TV-Dokumentation „Out of the ashes: Recovering the lost library of Herculaneum (Produced, Written and Directed by Julie Walker - a KBYU/BYU Television Production 2003)“, online abrufbar und Agnese Travaglione, „Verkohlte Papyrus-Rollen. Die antike Bibliothek der Villa dei Papiri und ihre Entzifferung, “ in Mühlenbrock und Richter (Hgg.), Verschüttet vom Vesuv (wie Anm. 23), (11-122), 120.

117 Vgl. Delattre, rouleaux d'Herculanum (wie Anm. 5), 113-116. 
MSI ist, dass die Papyri nun von anderen Orten der Welt als Neapel gleichsam „am Original“ studiert und digital aufbereitet werden könne, etwa durch visuelle Computerprogramme. Allerdings sind die MSI unter keinen Umständen als detailgetreue Kopien des Originals misszuverstehen und eine Autopsie in Neapel ist weiterhin geboten, da die komplexe dreidimensionale Stratigraphie der Papyri (siehe 6.2) auf den zweidimensionalen MSI mitunter irreführend wiedergegeben ist. Löcher oder Schatten von aufgewellten Teilen erscheinen teils fälschlich als Tinte und auch Strukturen wie Voluten können auf den MSI nur unzureichend erkannt werden. MSI und Autopsie ergänzen sich, ersetzen aber einander nicht. ${ }^{118}$ Papyrologen sind folglich seit rund 20 Jahren nicht zuletzt damit beschäftigt alte Ausgaben bereits aufgerollter Papyri auf Basis der MSI zu revidieren, meist mit erheblichem Neugewinn an Erkenntnis und substantiell verbesserten Texten.

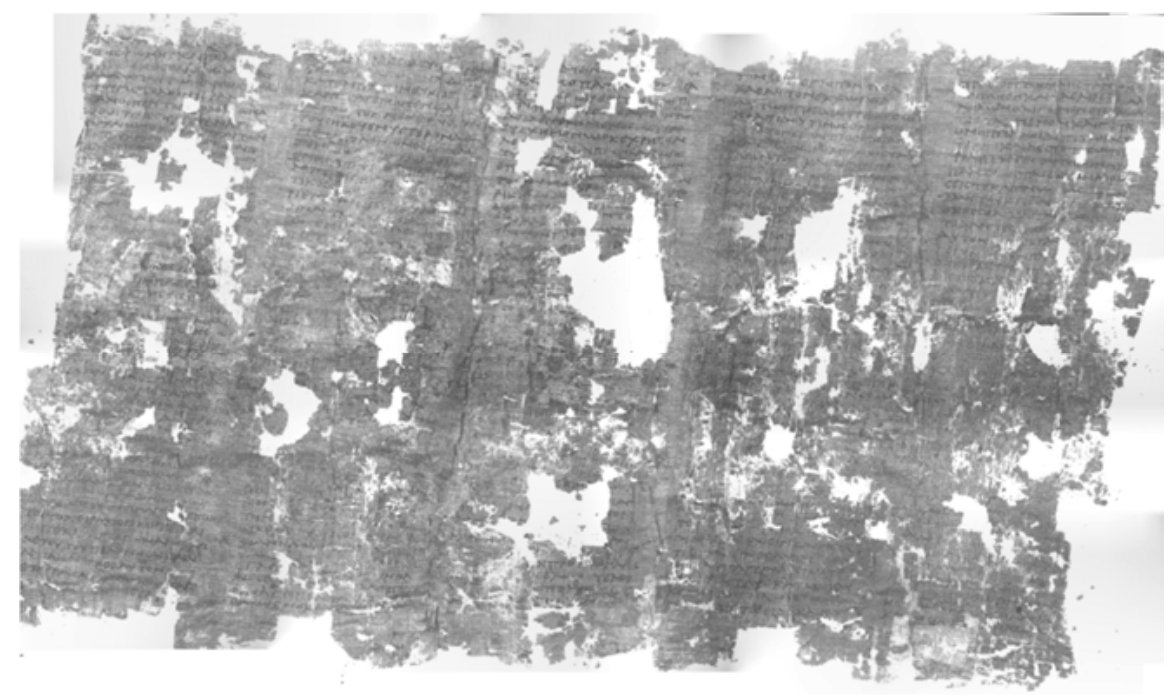

Abb. 29: Multispektralbild (MSI)- cornice 4 (=Kol. 15-19) von PHerc. 1021 (Philodems Index Academicorum)

118 Janko, How to read (wie Anm. 5), 124-126. 


\subsection{Reflectance Transformation Imaging (RTI-2014), 3D- Imaging und Analyse der Tinte}

In geringerem Umfang wurde auch nahinfrarot Reflectance Transformation Imaging (NIR-RTI) an den Papyri um die Protagonisten Jürgen Hammerstaedt und Kathryn Piquette erprobt (2014). An einzelnen Fragmenten wurde die Technik angewendet, welche, vereinfacht gesprochen, „plastischere“ Bilder als die MSI liefert, da der Papyrus von verschiedenen Winkeln aus fotografiert wird. Es entsteht ein „dreidimensionaler“ Eindruck und die Auflösung ist wesentlich höher als auf den MSI. Die Technik könnte somit insbesondere beim digitalen Erkennen von Sovrapposti und Sottoposti und für andere Fragen hilfreich sein, wobei der Kontrast zwischen Tinte und Papyrus nicht erheblich stärker als auf den MSI erscheint. Im Jahr 2016 hat ein Team um Brent Seales erstmals für den in England aufbewahrten PHerc. 118 ein komplettes 3D-Faksimilie eines Herkulanischen Papyrus angefertigt. Technik und Details variieren im Vergleich zu RTI, die Ergebnisse aber sind in einigen Punkten vergleichbar (Plastizität). ${ }^{119}$

Auch die Zusammensetzung der Tinte, welche eine gewisse Relevanz für das virtual unrolling hat (11.2), wurde während der letzten 5 Jahre in einigen Studien untersucht. ${ }^{120}$ Es stellte sich heraus, dass die Tinte der Herkulanischen Papyri offenbar nicht rein karbonbasiert ist, sondern auch metallische Elemente, besonders Blei, enthält. Der chemische Fußabdruck der Tinte könnte bei der Zuweisung von Papyrusfragmenten helfen.

119 Kathryn Piquette, „Illuminating the Herculaneum Papyri: Testing new imaging techniques on unrolled carbonised manuscript fragments, “ Digital Classics Online 3,2 (2017): (80-102); Brent Seales, „Visualizing PHerc. 118,“ Thinking 3D (2019) - T3D2019_INA.

120 Emmanuel Brun/Marine Cotte/Jonathan Wright/Maria Ruat/Pieter Tack/Laszlo Vincze/Claudio Ferrero/Daniel Delattre/ Vito Mocella, „Revealing Metallic Ink in Herculaneum Papyri,“ Proceedings of the National Academy of Sciences 14.113 (2016): (3751-3754); Pieter Tack/Marine Cotte/Stephen Bauters/Emmanuel Brun/Dipanjan Banerjee/Wim Bras/Claudio Ferrero/Daniel Delattre/Vito Mocella/Laszlo Vincze, „Tracking Ink Composition on Herculaneum Papyrus Scrolls: Quantification and speciation of lead by X-ray based techniques and Monte Carlo simulations,“ Scientific Reports 6 (2016); Olivier Bonnerot/Gianluca Del Mastro/Jürgen Hammerstaedt/Vito Mocella/Ira Rabin, „XRF ink analysis of some Herculaneum papyri,“ ZPE 216 (2020): (50-52). Überblick bei Vincenzo Damiani, „Ink in Herculaneum: A Survey of Recent Perspectives, “ in Traces of Ink (hg. von Lucia Raggetti; Leiden, 2021), (57-69). 


\subsection{Hyperspektralbilder (HSI: 2019)}

Als letzter großer technischer Fortschritt bei der Erforschung schon geöffneter Papyri kann eine Technik gelten, an deren experimentierweiser Anwendung ich 2016-2018 in meiner Zeit in Neapel selbst beteiligt war. ${ }^{121}$ Um die heute nicht mehr sichtbare Rückseite von Philodems „Geschichte der Akademie“ (Index Academicorum) - der Papyrus ist einer der wenigen Opisthographen der Sammlung (siehe 15.1), was bedeutet, dass er auch auf der Rückseite beschrieben ist sichtbar zu machen, war ein internationales Team (CNR/CNRS/Musée national d'histoire naturelle, Paris) im Februar 2018 für eine Woche in der Officina dei papiri, um Hyperspektralbilder (Hyperspectral images = HSI) des Papyrus zu machen. Diese wurden bis 2019 digital aufbereitet und werden philologisch für die editio maior des Index Academicorum immer noch ausgewertet. ${ }^{122}$ Primäres Ziel war das Durchröntgen der Vorderseite (Rekto) des Papyrus, um den Text auf der Rückseite (Verso) sichtbar zu machen. Bevor der Papyrus auf die Pappunterlage (cornice) geklebt wurde (von der er heute ohne größere Zerstörungen nicht ablösbar ist), hatte man auch die 12 Kolumnen des Verso abgezeichnet (Oxforder Disegni). Anhand eines gespiegelten „Lochabgleichs“ der Disegni mit dem Original war es mir möglich, die Versokolumnen mit einiger Sicherheit relativ zum Rekto zu lokalisieren. ${ }^{123} \mathrm{Da}$ aber die Disegni oft fehlerhaft oder unvollständig sind (6.3) und womöglich nicht alle Kolumnen auf dem Verso abgezeichnet wurden, war es ein Desiderat, den Text des Verso lesbar zu machen, zumal die Vermutung im Raum stand, dass einige der Nachträge oder Notizen auf dem Verso dieser Entwurfsfassung sogar von Philodems eigener Hand geschrieben worden sein könnten.

121 Marie Curie Individual Fellowship - 703798-Acad Hist: Philodemus' History of the Academy: Groundwork for a New Innovative Critical Edition. Für Details siehe https://www.iliesi.cnr.it/philodemus/.

122 Vgl. Aurelie Tournié/Kilian Fleischer/Inna Bukreeva/Francesca Palermo/Michela Perino/Adele Cedola/Christine Andraud/Graziano Ranocchia, „Ancient Greek text concealed on the back of unrolled papyrus revealed through shortwave-infrared hyperspectral imaging,“ Science Advances 5/10 (2019) - https://advances.sciencemag.org/content/5/10/eaav8936. Dabei wurde insbesondere auf das Programm der MOLAB platform (mobile Laboratorien) zurückgegriffen (IPERION CH grant agreement no.654028). Die Bilder wurden in den folgenden Monaten und Jahren ausgewertet (siehe auch 15).

123 Kilian Fleischer, „Die Lokalisierung der Verso-Kolumnen von PHerc. 1021,“ ZPE 204 (2017): (27-39). 

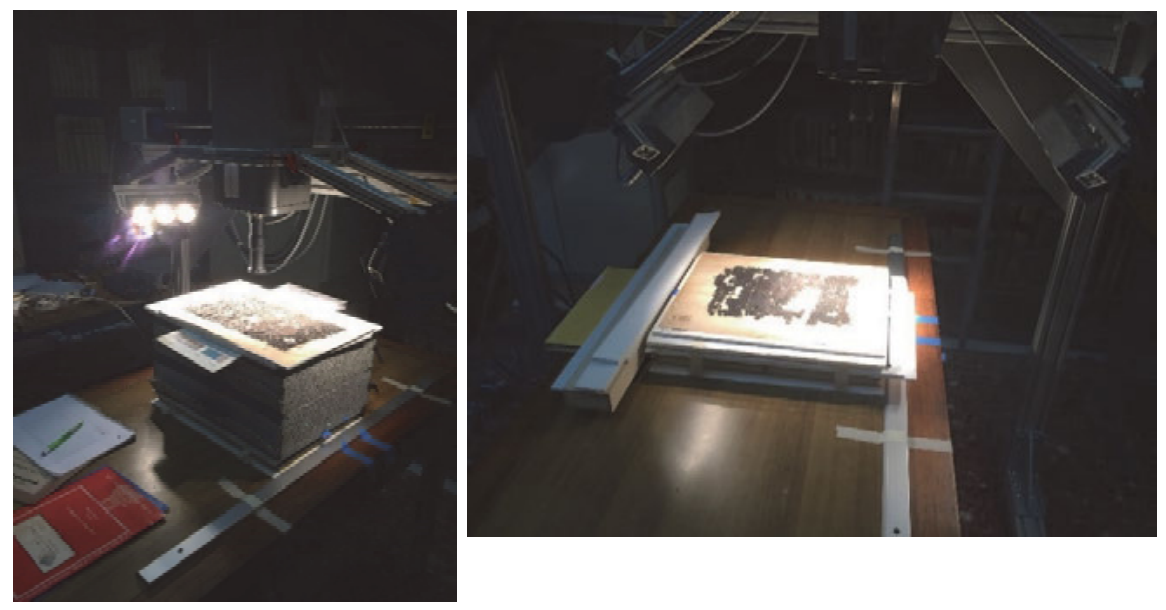

Abb. 30 und 31: Experimente 2018 mit Hyperspektraltechnik an PHerc. 1021 (Philodems Index Academicorum)

Dieses Ziel wurde teilweise erreicht, insofern tatsächlich hinter mehreren unbeschriebenen Teilen des Rekto (Interkolumnium) der Text des Verso sichtbar wurde, der sich auch mit den vor 220 Jahren gemachten Abzeichnungen deckt. Jedoch war es bisher nicht möglich, ${ }^{124}$ den Text auf der Rückseite zu lesen, wo dieser mit dem Text auf der Vorderseite überlappt. Ferner fanden sich von einigen Verso-Kolumnen keine Spuren, was vielleicht mit der unterschiedlichen Dicke oder Karbonisation von Teilen des Papyrus erklärt werden könnte. In einem Bereich ist auch Text sichtbar, wo die Disegni dies nicht vermuten lassen. ${ }^{125}$

124 Im Zuge des DFG-Projektes „Philodems Geschichte der Akademie (Index Academicorum)“ und des ERC-advanced Grant „GreekSchools“ laufen die Auswertungen der HSI noch, d.h. dass Physiker/Informatiker die Rohdaten nochmals aufbereiten, um die „störende“ Vorderseite herauszufiltern.

125 Im Mai 2018 wurde ohne nennenswerten Erfolg, ebenfalls über die MOLAB platform (vgl. Anm. 122), für einige Tage noch TeraHerz-Imaging am Index Academicorum erprobt (mit dem Ziel das Verso zu lesen). Jedoch mag hier der konkrete Versuchsaufbau nicht optimal gewesen sein, so dass diese Technik nicht per se ungeeignet ist. 


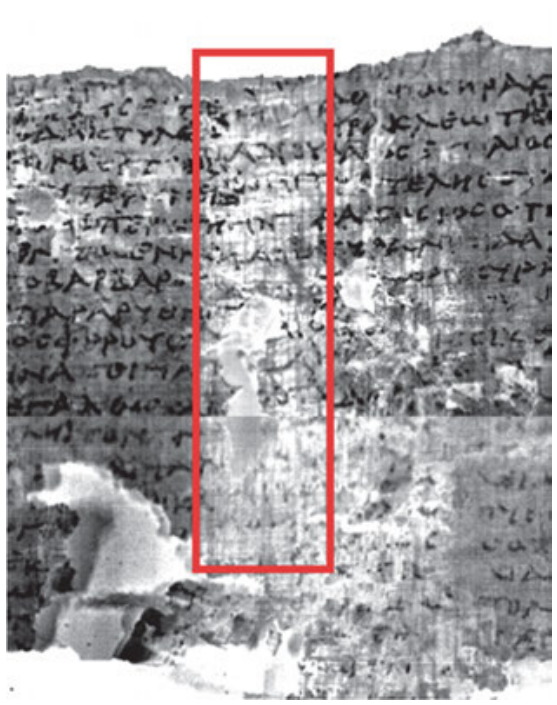

Abb. 32: Text des Verso-spiegelverkehrt

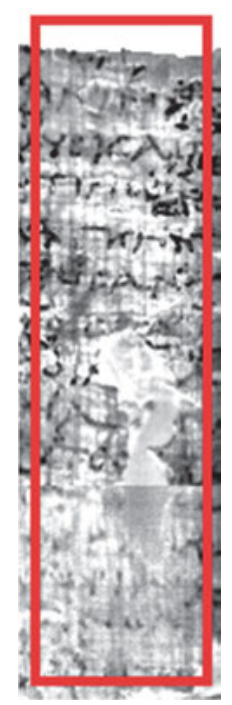

Abb. 33: Text des Verso

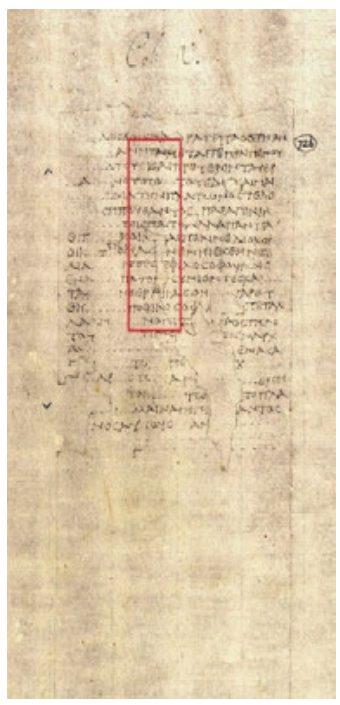

Abb. 34: Stelle im Disegno

Von weitaus größerer Bedeutung war nun aber ein überraschendes Nebenergebnis: Die Hyperspektralbilder bieten auf dem Rekto einen wesentlich besseren Kontrast als die MSI. Die HSI haben es mir erlaubt den schon auf Basis der Multispektralbilder um $20 \%$ vermehrten bzw. verbesserten Text des Index Academicorum noch einmal um 5-10\% zu vermehren, d.h. viele neue Informationen zur Akademie zu gewinnen. Vermutlich ist es lohnenswert, von der gesamten Herkulanischen Sammlung - also von allen schon geöffneten Papyri 20 Jahre nach den MSI auch HSI zu machen. Die HSI zeigen Text an Stellen, wo man weder mit dem bloßen Auge noch auf den MSI etwas erkennt. Jedoch sind die HSI den MSI nicht an jeder Stelle überlegen, so dass sie im Vergleich mit den MSI (neben Autopsie und Disegni) genutzt werden sollten.

Zur technischen Seite sei vermerkt, dass die HSI mit 1000-2500 nm einen wesentlich größeren Wellenlängenbereich als die MSI abdecken. Die genaue Bezeichnung der angewandten Technik ist shortwave infrared hyperspectral imaging (SWIR-HSI). Die Rohdaten wurden mittels einer Hauptkomponentenanalyse (PCA) anhand der PC1 und PC3-Achse statistisch aufbereitet, was einen starken Kontrast ergibt. Die Auflösung ist weniger hoch als auf den MSI, was aber durch einen einfachen Wechsel der Kameralinse behoben werden kann. Pro cormice wurden vier Durchgänge von jeweils ca. 20-30 Minuten benö- 
tigt, so dass die Bilder „gestitched“ sind. Die HSI-Experimente am Index Academicorum wurden in effektiv nur 3 Tagen durchgeführt. Bei weiteren Experimenten wäre das Verfahren in Kürze hinsichtlich verschiedener Aspekte optimierbar, so dass die komplette Sammlung in zeitlich vertretbarem Rahmen mit Hyperspectral Imaging fotografiert werden könnte.

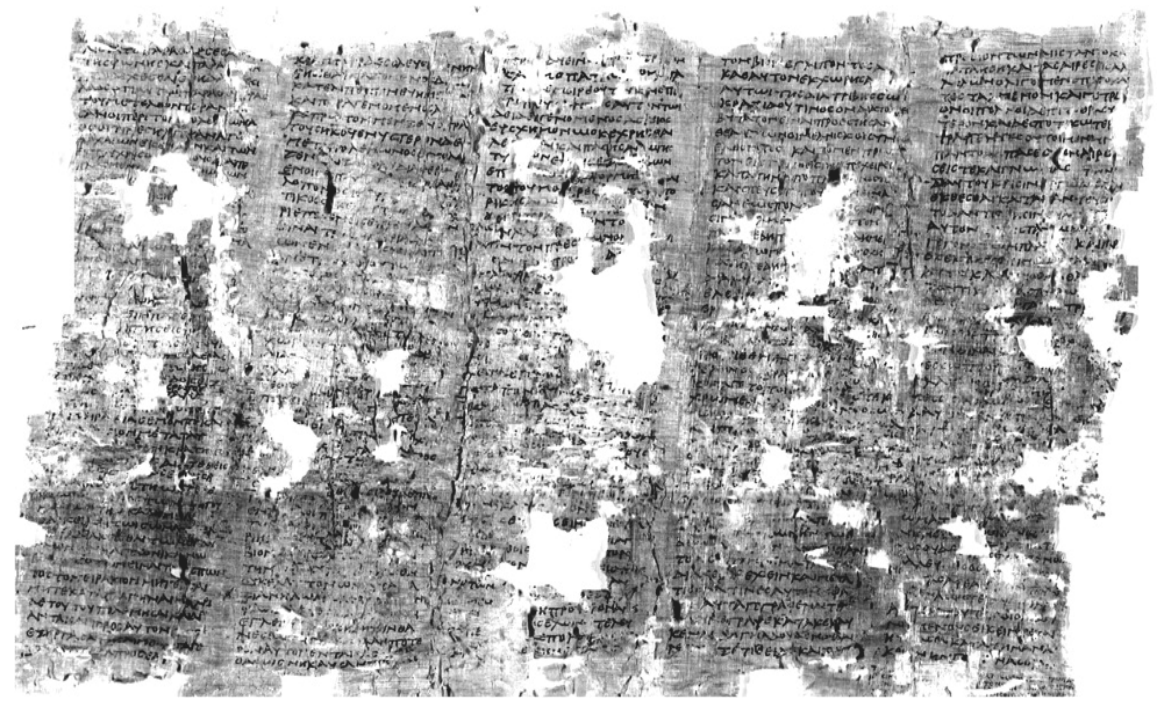

Abb. 35: Hyperspektralbild (HSI)- cornice 4 (=Kol. 15-19) von PHerc. 1021 (Philodems Index Academicorum)

\subsection{Künftige Experimente an geöffneten Papyri und das „Durchröntgen“ von Sovrapposti}

Im Rahmen des EU-geförderten ERC-Projektes „GreekSchools“ unter dem Principal Investigator Graziano Ranocchia ${ }^{126}$ sollen innerhalb der nächsten Jahre (2021-2025) durch mobile Laboratorien der European Infrastructure for Heritage Science nochmals in modifizierter Weise Shortwave-Infrared Hyperspectral

126 ERC Advanced Grant 885222-GreekSchools - The Greek Philosophical Schools according to Europe's earliest „history of philosophy“ - Towards a new pioneering critical edition of Philodemus' Arrangement of the Philosopher. Das Projekt (ca. 2,5 Mio Euro) ist an der Universität Pisa angesiedelt und schließt als Kooperationspartner etwa das CNR Institute of Cultural Heritage, das CNR Institute for Computational Linguistics und die Biblioteca Nazionale di Napoli (BNN) ein. 
Imaging und High-resolution TeraHertz Imaging sowie High-resolution X-Ray Fluorescence Mapping ${ }^{127}$ für das Lesen des Verso des Index Academicorum (und anderer Papyri) genutzt werden.

Die schon angesprochene Stratigraphie der Papyri, übereinanderliegende Lagen (Sovrapposti, teils Doppel-Sovrapposti oder gar Dreifach- bzw. VierfachSovrapposti), stellen ein besonderes Problem der Herkulanischen Papyrologie dar. Im Zuge obengenannten Projektes soll daher auch versucht werden, durch diese Sovrapposti „hindurchzuröntgen“ und den darunterliegenden Text virtuell sichtbar zu machen. Letztlich ist dieses „Lagen-Durchröntgen“ in technischer Hinsicht aufs Engste mit dem Lesen des Verso eines Papyrus verbunden, so dass alle oben genannten Techniken auch mutatis mutandis bei dieser Problemstellung Anwendung finden sollen. Daneben werden OberflächenProfilometrie mittels hochauflösenden 3D-Mikroskopen ${ }^{128}$ sowie TiefenProfilometrie mittels Kernspinresonanz-Relaxometrie (NMR-relaxometry) und Optische Kohärenztomographie für das Durchleuten der Lagen getestet. Ein flächendeckendes, standardisiertes „Durchröntgen“ aller Sovrapposti in Herkulanischen Papyri würde einen nicht zu unterschätzenden Textzuwachs liefern. Bei derlei Experimenten ist immer schwer vorherzusagen, welche Technik oder Technikkombination die gewünschten Resultate liefert. Zuletzt sei auch nicht unerwähnt, dass es noch unter vielen PHerc.-Nummern aufgerollte Papyri gibt, die noch niemals ediert wurden. Oftmals handelt es sich nur um kleinere oder schlechtere Stücke, aber mitunter sind auch größere, vergleichsweise gut lesbare Fragmente darunter.

127 High-resolution X-Ray Fluorescence wurde für die Oberfläche bereits von Brun et al., „Revealing Mettalic Ink“ (wie Anm. 120) angewandt.

128 Oberflächen-Profilometrie, jedoch mit anderem Ansatz, wurde mit ansprechenden Resultaten schon von Seales (wie Anm. 119) an einem Herkulanischen Papyrus getestet (siehe 10.3). Seales will in den nächsten Jahren auch Photogrammetrie auf die gesamte Sammlung anwenden. 